\title{
ANÁLISE PARASITOLÓGICA DE AMBIENTES UTILIZADOS PARA RECREAÇÃO INFANTIL
}

\author{
Karina Rodrigues dos Santos ${ }^{1}$, Amanda Marques da Silva ${ }^{2}$, Severino \\ Cavalcante de Sousa ${ }^{1}$, Adriana do Nascimento Sousa Farias ${ }^{3}$; Janilda Barros \\ Santiago Oliveira ${ }^{3}$
}

1 Prof. Dr. Departamento de Medicina, CMRV/UFPI, Campus Ministro Reis Velloso, Parnaíba, Piauí - Brasil.

${ }^{2}$ Discente do curso de Biomedicina da Universidade Federal do Piauí-UFPI Campus Ministro Reis Velloso, Parnaíba, Piauí- Brasil.

${ }^{3}$ Veterinária da Universidade Federal do Piauí (UFPI), Bom Jesus, Piauí - Brasil.

* Author for correspondence: Karina Rodrigues dos Santos. UFPI. Parnaíba, Piauí,

Brasil. E-mail: krsantos2004@yahoo.com.br

\section{Recebido em: 06/04/2019 - Aprovado em: 10/06/2019 - Publicado em: 30/06/2019 DOI: 10.18677/EnciBio_2019A154}

\begin{abstract}
RESUMO
As enteroparasitoses são consideradas um grande problema de saúde pública, afetando principalmente a população infantil entre zero a 10 anos de idade. A contaminação acontece através da água e alimentos contaminados, entretanto em alguns casos pode acontecer através de ambientes onde estão presentes fezes que contenham ovos ou cistos dos parasitos. O objetivo deste trabalho foi verificar a presença de ovos de parasitos gastrointestinais no solo de ambientes freqüentados por crianças na cidade de Bom Jesus-PI, Brasil. Através dos métodos de Hoffman, Faust e técnica de Willis Molley, foram analisadas 60 amostras de solo coletadas em 10 ambientes com áreas de recreação, sendo que uma amostra era utilizada na preparação das três técnicas, totalizando 180 exames. Através das análises, verificou-se que $97,78 \%$ das amostras foram negativas para qualquer espécie de parasito, sendo que apenas $2,22 \%$ das amostras foram positivas para cistos de Giardia spp. que pode ser explicado pela grande resistência que essa forma parasitária possui e pelo fato dos ambientes envolvidos serem freqüentados por crianças de baixa idade, considerada a população mais acometida pelas enteroparasitoses.
\end{abstract}

PALAVRAS-CHAVE: crianças; contaminação do solo, enteroparasitoses.

\section{PARASITOLOGICAL ANALYSIS OF ENVIRONMENTS USED FOR CHILD}

\begin{abstract}
Enteroparasitoses are considered to be a major public health problem, affecting mainly the child population between 0 and 10 years of age. Contamination occurs through contaminated water and food, however in some cases it may occur through environments where feces containing eggs or parasitic cysts are present. The objective of this work was to verify the presence of eggs of gastrointestinal parasites in the soil of environments frequented by children in the city of Bom Jesus-PI, Brazil.
\end{abstract}


Through the methods of Hoffman, Faust and Willis Molley technique, 60 soil samples were analyzed in 10 environments with recreation areas, and a sample was used in the preparation of the three techniques, totaling 180 exams. Through the analyzes, it was verified that $97.78 \%$ of the samples were negative for any species of parasite, and only $2.22 \%$ of the samples were positive for Giardia spp. which can be explained by the great resistance that this parasitic form has and by the fact that the environments involved are frequented by children of low age, considered the population most affected by enteroparasitoses.

KEYWORDS: Children; Enteroparasitoses; Ground contamination.

\section{INTRODUÇÃO}

As enteroparasitoses são doenças causadas por parasitos intestinais, como helmintos ou protozoários, que podem levar a alterações no estado físico, psicológico e social da pessoa infectada (MELO et al., 2014), sendo consideradas um importante problema de saúde pública, contribuindo para a morbidade de pessoas em todo o mundo (ABREU et al., 2014).

A prevalência das doenças parasitárias em um país apresenta variações de acordo com seu desenvolvimento e infraestrutura (BUSATO et al., 2014), sendo mais prevalentes em países em desenvolvimento que apresentam precárias condições de saneamento básico, de higiene, baixo nível socioeconômico e baixo grau de escolaridade (MELO et al., 2014).

As enteroparasitoses podem causar problemas na qualidade de vida dos portadores, principalmente em crianças que em geral são as mais acometidas por essas infecções (ABREU et al., 2014; MORI et al., 2016). Essa prevalência em crianças se deve ao fato da imunidade não estar completamente desenvolvida, sendo insuficiente para que ocorra a eliminação dos parasitos (CAVAGNOLLI et al., 2015). Outro fator que torna as crianças mais susceptíveis as infecções por parasitos gastrointestinais é o hábito de higiene inadequada, pois como se trata de infante, as informações necessárias para se manter uma boa higiene ainda não são completamente compreendidas (PRITSCH; FRIGHETTO, 2016). As manifestações clínicas causadas pelas doenças parasitárias variam entre: diarréia, colites, má absorção intestinal, anemia, obstrução intestinal, desnutrição, deficiência no aprendizado e no desenvolvimento físico da criança, dependendo de qual parasito está causando a infecção (ANDRADE et al., 2015).

A transmissão das enteroparasitoses acontece de forma oral-fecal, através da ingestão de ovos de helmintos ou cistos de protozoários presentes nos alimentos e água contaminadas com fezes (MASCARENHAS; SILVA, 2016). Em alguns casos o contato com os ovos ou cistos dos parasitos acontece em ambientes que fazem parte da rotina da criança, como por exemplo, áreas de recreação em escolas, creches, parques e áreas residenciais, que não possuem tratamento ou limpeza apropriada do solo (areia), ou que representam locais impróprios para o descarte de dejetos de pias e armazenamento dos dejetos sanitários (MIRANDA et al., 2015; BALDINI et al., 2015; PRITSCH; FRIGHETTO, 2016).

O diagnóstico e tratamento das enteroparasitoses são extremamente importantes para a diminuição do número de casos de pessoas infectadas (ANDRADE et al., 2015), entretanto, se não extintas as fontes de contaminação os números continuarão constantes, pois ao mesmo tempo em que pessoas infectadas estão sendo diagnosticadas e tratadas, outras estarão sendo contaminadas com algum tipo de parasito gastrointestinal (SOUSA et al., 2014).

Tendo em vista todos os fatores expostos e a alta frequência de enteroparasitoses no Brasil, o objetivo deste estudo foi verificar a presença de ovos 
de helmintos ou cistos de protozoários ou larvas de parasitos gastrintestinais no solo de instituições frequentadas por crianças, na cidade de Bom Jesus - Piauí, Brasil.

\section{MATERIAL E MÉTODOS}

Foram escolhidos 10 ambientes frequentados por crianças e que possuíam área de recreação, dentre eles, três escolas particulares, que foram enumeradas de $1,2,3$, no intuito de preservar a identidades dos locais envolvidos, cinco escolas municipais, enumeradas de $4,5,6,7,8$, que possuíam crianças matriculadas entre três a 10 anos de idade, uma creche, enumerada de 9 e um clube de lazer da região identificado como 10. Todos esses ambientes estão localizados no município de Bom Jesus, situado a 635 km da capital Teresina, no Estado do Piauí.

Além das análises das areias das escolas, os supervisores foram indagados sobre o acesso de animais aos ambientes, se foi utilizado algum produto na areia e se houve casos de doenças parasitárias dentre os alunos destas escolas. Todas as instituições que fizeram parte deste estudo foram identificadas e os dados referentes a estas, anotados em fichas individuais.

Foram coletadas amostras de areia de cada instituição no período entre setembro a dezembro de 2010 e maio a julho de 2011, acondicionadas em caixas e mantidas à $10^{\circ} \mathrm{C}$. As coletas de areia foram realizadas em três pontos diferentes de cada instituição, em cada ponto foram colhidas duas amostras, uma superficial e uma profunda, com o auxílio de um Becker.

As amostras foram enviadas ao laboratório de Parasitologia da Universidade Federal do Piauí. Para análise das amostras foram utilizadas as técnicas de sedimentação espontânea para avaliar ovos de nematódeos, cestódeos e trematódeos, a técnica de Faust (HOFFMAN, 1987) para a procura de cisto de Giardia spp. e a técnica de Willis Molley (HOFFMAN, 1987) para ovos leves de nematódeos.

Foram coletadas 50 gramas de solo (areia), desta quantidade $5 \mathrm{~g}$ foram utilizados para a técnica de willis, $1 \mathrm{~g}$ para a técnica de Faust e $20 \mathrm{~g}$ para a técnica de sedimentação espontânea. Após o preparo das técnicas, as amostras foram analisadas com auxílio de microscópio óptico em aumento de 10x e para confirmação dos achados foi utilizada a objetiva de 40x.

\section{RESULTADOS E DISCUSSÃO}

No período em que se sucedeu o estudo foram coletadas seis amostras de locais diferentes dos 10 ambientes envolvidos, totalizando 60 amostras de solo. Foram preparadas três lâminas de cada amostra para a realização dos exames coproparasitológicos, citados anteriormente, totalizando 180 exames cujos resultados revelaram que somente $2,22 \%$ das amostras do solo estavam contaminadas com cistos de Giardia spp. e estes foram os únicos achados após as análises das amostras, sendo os exames coproparasitológicos negativos para outros tipos de ovos e cistos de parasitos (Tabela 1). 
TABELA 1: Principais parasitos gastrointestinais investigados em amostras de areia de recreação infantil no período de setembro a dezembro de 2010 e maio a julho de 2011, no município de Bom Jesus-PI.

\begin{tabular}{ll}
\hline Parasito Intestinal & Prevalência (\%) \\
\hline Ancylostoma spp. & 0 \\
Ascaris lumbricoides & 0 \\
Enterobius vermicularis & 0 \\
Giardia spp. & $2,22 \%$ \\
Toxocara spp. & 0 \\
Trichuris trichiuria & 0 \\
\hline
\end{tabular}

Este resultado foi semelhante ao encontrado por Sousa et al. (2014) em areias das praias urbanas de João Pessoa/PB, estes encontraram uma porcentagem de $9,7 \%$ de amostras positivas para cistos de Giardia spp. sendo observado neste estudo que a presença de umidade ou a falta desta não tinha influencia na identificação deste protozoário, demonstrando uma grande resistência dos cistos de Giardia spp. ao ambiente externo.

Segundo Saito e Rodrigues (2012) os cistos de Giardia spp. possuem grande resistência e proteção as mais variadas variações de umidade, produtos químicos e a temperatura, devido a parede cística ser composta de uma camada interna membranosa formada por três proteínas diferentes (CWP1, CWP2, CWP3) e por carboidratos, dentre os quais se destaca a N-acetilgalactosamina,e a camada exterior ser protofilamentosa. Através de uma pesquisa realizada por Franco (2007) foi comprovado que os cistos de Giardia spp. também possuem resistência a cloração da água e possuem persistência às condições ambientais, podendo permanecer infectantes por até seis meses no ambiente, a uma temperatura de $20^{\circ} \mathrm{C}$.

Após a coleta de amostras de areias das diferentes escolas foram realizadas observações dos diferentes ambientes (Tabela 2) e consultas aos supervisores das Escolas sobre as áreas de recreação, sendo possível obter as seguintes informações:

A área de recreação da escola 1 era toda murada, de difícil acesso para animais e fácil acesso para as crianças. A areia coletada não passou por nenhum tratamento e nenhuma criança apresentou lesão cutânea ou o responsável não teve conhecimento.

Após a observação da escola 2 foi possível averiguar que a área de recreação possuía muros e uma proteção com tela ao redor dos brinquedos, favorecendo o acesso somente das crianças, os animais não possuem acesso a esta área. A areia do local não recebeu nenhum tratamento e segundo a responsável pelo estabelecimento uma criança e um adolescente apresentou Larva Migrans cutânea.

A área de recreação da escola 3 era toda cercada por muros. A areia não recebeu tratamento e não teve nenhum relato de crianças com Larva Migrans cutânea.

Os ambientes das escolas 4 e 5 possuíam área cercada com livre acesso de crianças e também de várias espécies de animais. Nenhuma criança apresentou Larva Migrans cutânea e a areia coletada não passou por nenhum tipo de 
tratamento. As areias de recreação das escolas 6 e 7 eram muradas, os animais não possuem acesso, somente crianças. A areia não recebeu tratamento e nenhuma criança apresentou lesão cutânea.

O ambiente 8 possuía áreas de recreação muradas sem acesso para animais, somente crianças possuem acesso. A areia não recebeu qualquer tratamento e segundo relato da diretoria da escola, cinco crianças apresentaram Larva Migrans cutânea durante o período que decorreu este estudo. No ambiente 9 a área de recreação é murada, a areia não recebeu nenhum tratamento e nenhuma criança apresentou lesão cutânea.

Por último, o ambiente 10 possui área de lazer murada, sendo uma área ampla onde pessoas de várias idades têm acesso a esse local. O responsável relatou que a areia é tratada por aspersão com água sanitária e nenhuma criança apresentou lesão cutânea neste período.

As amostras positivas foram provenientes dos ambientes 4 e 7 (Tabela 2), ou seja, escolas públicas, sendo que o ambiente 4 é cercado, mas é acessado tanto por crianças como pelos animais, sugerindo que o solo é propício a presença de helmintos e protozoários, devido ao fato desses animais defecarem na areia, assim como as crianças que podem contaminar a areia com resíduos de fezes contendo esses parasitos, sendo esse um dos motivos para o achado de cisto de Giardia spp.

O ambiente 7 possui área murada, todavia, de acordo com as informações obtidas através dos supervisores, constatou-se que a areia não passou por nenhum tipo de tratamento e que os responsáveis não sabiam como realizar tal procedimento, sendo esse um dos motivos que pode ter levado a presença deste parasito no solo.

TABELA 2: Diferentes ambientes, resultados obtidos através das análises das amostras de solo, demonstrando o local, profundidade e qual técnica utilizada na identificação do parasito presente na amostra.

\begin{tabular}{|c|c|c|c|c|c|c|c|}
\hline Escolas & $\begin{array}{l}\text { Amostra } \\
{ }^{*} \text { D.S. }\end{array}$ & $\begin{array}{l}\text { Amostra } \\
{ }^{*} \text { D.P. }\end{array}$ & $\begin{array}{l}\text { Amostra } \\
{ }^{*} \text { C.S. }\end{array}$ & $\begin{array}{l}\text { Amostra } \\
{ }^{*} \text { C.P. }\end{array}$ & $\begin{array}{l}\text { Amostra } \\
{ }^{*} \text { E.S. }\end{array}$ & $\begin{array}{l}\text { Amostra } \\
{ }^{*} \text { E.P. }\end{array}$ & $\begin{array}{l}\text { Técnica } \\
\text { utilizada na } \\
\text { identificação } \\
\text { dos } \\
\text { parasitos }\end{array}$ \\
\hline 1 & - & - & - & - & - & - & $\begin{array}{l}\text { Não foi } \\
\text { encontrado } \\
\text { em } \\
\text { nenhuma } \\
\text { técnica }\end{array}$ \\
\hline 2 & - & - & - & - & - & - & $\begin{array}{l}\text { Não foi } \\
\text { encontrado } \\
\text { em } \\
\text { nenhuma } \\
\text { técnica }\end{array}$ \\
\hline 3 & - & - & - & - & - & - & $\begin{array}{l}\text { Não foi } \\
\text { encontrado } \\
\text { em } \\
\text { nenhuma } \\
\text { técnica }\end{array}$ \\
\hline 4 & + & + & - & - & - & - & Hoffman \\
\hline
\end{tabular}




\begin{tabular}{|c|c|c|c|c|c|c|c|}
\hline 5 & - & - & - & - & - & - & $\begin{array}{l}\text { Não foi } \\
\text { encontrado } \\
\text { em } \\
\text { nenhuma } \\
\text { técnica }\end{array}$ \\
\hline 6 & - & - & - & - & - & - & $\begin{array}{l}\text { Não foi } \\
\text { encontrado } \\
\text { em } \\
\text { nenhuma } \\
\text { técnica }\end{array}$ \\
\hline 7 & - & + & - & + & - & - & Faust \\
\hline 8 & - & - & - & - & - & - & $\begin{array}{l}\text { Não foi } \\
\text { encontrado } \\
\text { em } \\
\text { nenhuma } \\
\text { técnica }\end{array}$ \\
\hline 9 & - & - & - & - & - & - & $\begin{array}{l}\text { Não foi } \\
\text { encontrado } \\
\text { em } \\
\text { nenhuma } \\
\text { técnica }\end{array}$ \\
\hline 10 & - & - & - & - & - & - & $\begin{array}{l}\text { Não foi } \\
\text { encontrado } \\
\text { em } \\
\text { nenhuma } \\
\text { técnica }\end{array}$ \\
\hline
\end{tabular}

${ }^{*}$ D.S. $=$ Direita superficial; ${ }^{*}$ D.P. $=$ Direita profunda; ${ }^{*}$ C.S. $=$ Central superficial; ${ }^{*}$ C.P. $=$ Central profunda; ${ }^{\star} E . S .=$ Esquerda superficial; ${ }^{*}$ E.P. $=$ Esquerda profunda

Um total de $97,78 \%$ das amostras foram negativas para qualquer espécie de parasito (Tabela 3).

TABELA 3: Porcentagem e número de contaminações das amostras de areia das instituições no período de setembro a dezembro de 2010, no município de Bom Jesus-PI.

\section{Amostras Positivas (\%) $\quad$ Amostras negativas (\%)}

$\begin{array}{ll}\mathrm{N}=2,22 & \mathrm{~N}=97,78\end{array}$

Esses resultados foram encontrados nos oito ambientes em que as amostras coletadas foram negativas, um dos motivos pode ser devido essas escolas serem muradas o que pode ter dificultado ou impedido a entrada de animais nas áreas de recreação, resultando na ausência de fezes desses animais na areia, outro fator que pode ter dificultado a prevalência desses parasitos no solo foi o tratamento da areia, sendo que um dos oito ambientes realizou o tratamento através da aspersão do solo com água sanitária, o que diminui ou impede a proliferação e conclusão do ciclo de 
vida dos parasitos gastrointestinais na areia (MELO et al., 2014; BALDINI et al., 2015; SAMPAIO; BARROS, 2017).

Segundo Oliveira et al. (2011) é comum encontrar resultados negativos para as amostras coletadas de locais que possuem barreira ou cercas de proteção nas áreas de recreação que impedem a entrada e acesso de animais, como cães e gatos, uma vez que essas proteções previnem a presença de parasitos gastrointestinais no solo, o que explica a ausência de helminto e protozoários no solo de ambientes que apresentaram muros ao redor das áreas de recreação neste estudo (ABREU et al., , 2014, ANDRADE et al., 2015).

A proteção do solo de áreas de recreação com lonas ou outro material, possui total importância na diminuição dos casos de pessoas contaminadas por helmintos presentes na areia. Segundo Oliveira et al. (2011) essas barreiras físicas dificultam a entrada dos animais e estão intimamente relacionadas a diminuição da contaminação ambiental por esses geohelmintos.

A elevada porcentagem de amostras negativas é considerada um ponto positivo, uma vez que foram realizados 180 exames de amostras coletadas entre setembro a dezembro de 2010 e maio a julho de 2011, o que equivale a sete meses de coletas, e se relacionar esses fatores com a falta de infraestrutura e saneamento básico no município de Bom Jesus a probabilidade de ter ausência de parasitos na maioria das amostras é baixa, demonstrando que mesmo na presença desses fatores agravantes os ambientes apresentam baixa prevalência de parasitos gastrointestinais no solo, deduzindo que a população infantil está possuindo hábitos higiênicos adequados e que os muros estão mantendo os animais longe dos locais de recreação freqüentados pelas crianças.

A ausência de ovos de nematódeos no presente estudo pode ser explicada pelo fato de a maioria das instituições serem muradas e de difícil acesso aos animais de rua e em conjunto pela época de coleta, que foi de setembro a dezembro caracterizado como período seco. Neste período seco a cidade de Bom Jesus apresenta baixa precipitação pluviométrica, o que faz com que os parasitos apresentem dificuldades em completarem os ciclos de vida parasitários, pois as temperaturas elevadas ao mesmo tempo em que aceleram o desenvolvimento larval (ovo até larva infectante) dos helmintos podem reduzir o tempo de sobrevivência das larvas desses no ambiente (ANDRADE et al., 2015; MIRANDA et al., 2015; PRITSCH; FRIGHETTO, 2016).

Neste trabalho, a prevalência de cistos de Giardia spp. no solo (areia) pode ser explicada tanto pela alta resistência ao ambiente que essa forma parasitária possui, como pelo fato de os locais de coleta das amostras serem áreas de recreação utilizadas por crianças de baixa idade, que segundo Miranda et al. (2015) e Mascarenhas e Silva (2016), são as mais susceptíveis a adquirir esses parasitos gastrointestinais, devido a imunidade não estar completamente desenvolvida e seus hábitos higiênicos inadequados, sendo que em alguns casos acabam contaminando a areia com suas próprias fezes, ou por defecarem na areia, ou por já chegarem ao local com suas roupas sujas com fezes contaminadas (SOUSA et al., 2014).

Essas informações corroboram com os achados de outros autores que afirmam que são comuns as infecções causadas por Giardia spp. em crianças entre a faixa etária de 0 a 10 anos, assim como é comum à presença destes parasitos em instituições como: escolas infantis, creches e áreas de recreação frequentadas por crianças, pois o contato pessoa-pessoa é freqüente e a higiene pessoal e coletiva é difícil de ser regulada (ABREU et al., 2014; MELO et al., 2014; SILVA et al., 2015; ANDRADE et al., 2015; CAVAGNOLLI et al., 2015; MASCARENHAS; SILVA, 2016). 
Além do mais, a cidade do Bom Jesus possui facilidade na disseminação das enteroparasitoses e na contaminação por estes parasitos gastrointestinais (Giardia spp.) devido ao fato da cidade apresentar saneamento básico inadequado e pela ausência de estações de tratamento de água, esgoto e solo, que segundo e Busato et al. (2014) e Silva et al. (2015) são alguns dos principais agravantes para o aumento da prevalências dos casos de enteroparasitoses (PRITSCH; FRIGHETTO, 2016; SAMPAIO, BARROS, 2017).

\section{CONCLUSÃO}

Neste estudo foi encontrado nos solos das diferentes instituições da cidade de Bom Jesus - Piauí, Brasil, somente cistos de Giardia spp. devido sua alta resistência no ambiente e devido à fatores climáticos da região, pois no período em que o estudo foi realizado a cidade de Bom Jesus apresenta uma temperatura alta e clima muito seco, sendo desfavorável para o desenvolvimento do ciclo de vida dos parasitos nematódeos. E concluímos também que os métodos de proteção e hábitos de higiene estão sendo executados de maneira eficaz pela população infantil e pelas instituições da cidade de Bom Jesus.

\section{REFERÊNCIAS}

ABREU, L. K; BRAGA, L. S.; NAVASCONI, T. R.; SILVA, R. C. R. Prevalência e aspectos sócio-epidemiológicos de enteroparasitoses em crianças do centro municipal de educação infantil em Janiópolis-PR. Revista Saúde e Biologia, v.9, n.3, p.76-84, 2014. Disponível em: <http://www.revista.grupointegrado.br/sabios>.

ANDRADE, A. L. F. J.; ARAÚJO, K. B. S; MEDEIROS, V. S. Ocorrência de parasitos com potencial zoonótico em fezes de cães coletadas em vias públicas da cidade de Natal. Revista Humano Ser, v.1, n.1, p. 52-59, 2015. Disponível em: $<$ https://periodicos.unifacex.com.br/humanoser/article/download/624/143>.

BALDINI, C. L.; CRUZ, L. K. S.; LEMES, S. R.; VALE, M. A. A. B.; BARBERI, M.; RUBIN J. C. R.; MELO-REI, P. R. Parasitos na área recreativa infantil do parque vaca brava, Goiânia - GO. Estudos, Goiânia, v.42, n.4, p. 575-582, 2015. Disponível

em:

<https://seer.pucgoias.edu.br/index.php/estudos/article/viewFile/4377/2509>.

BUSATO, M. A.; ANTONIOLLI, M. A.; TEO, C. R. P. A.; FERRAZ, L.; POLI, G.; TONINI, P. Relação de parasitoses intestinais com as condições de saneamento básico. Revista Ciência, Cuidado e Saúde, v.13, n.2, p. 357-363, 2014.

Disponível em: $<$ https://www.researchgate.net/publication/280978868_Relacao_de_parasitoses_inte stinais_com_as_condicoes_de_saneamento_basico>.

CAVAGNOLLI, N. I.; CAMELLO, J. T.; TESSER, S.; POETA, J.; RODRIGUES, A. D. Prevalência de enteroparasitoses e análise socioeconômica de escolares em Flores da Cunha-RS. Revista de Patologia Tropical, v. 44, n.3, p. 312-322, 2015. Disponível em: <https://revistas.ufg.br/iptsp/article/download/38018/19152/>. doi: $10.5216 /$ rpt.v44i3.38018

FRANCO, R. M. B. Protozoários de veiculação hídrica. Revista Panamericana de Infectologia, v. 9, n.1, p. 36-43, 2007. Disponível em: 
$<$ http://www.educadores.diaadia.pr.gov.br/arquivos/File/2010/artigos_teses/2011/cien cias/03protozoarios_veiculacao_hidrica.pdf>.

GRACILIANO, J. J. N.; FARIAS, J. A. C.; ROCHA, T. J. M. Contaminação da areia por parasitos de importância humana detectados nas praias da orla marítima de Maceió-AL. Arquivos Médicos dos Hospitais da Faculdade de Ciências Médicas da Casa de São Paulo, v. 62, n. 2, p. 81-84, 2017. Disponível em: <http://arquivosmedicos.fcmsantacasasp.edu.br/index.php/AMSCSP/article/view/43>

HOFFMAN, R. P. Diagnóstico de parasitismo veterinário. Porto Alegre: Sulina, 1987. p. 33-39.

MASCARENHAS, J. P.; SILVA, D. S. Presença de parasitos no solo das áreas de recreação em escolas de educação infantil. Journal of Nursing and Health , v.1, n.1, p. 76-82, 2016. Disponível em: $<$ https://periodicos.ufpel.edu.br/ojs2/index.php/enfermagem/article/view/5732>.

MELO, A. C. F. L.; CEIA, E. A. J.; AZEVEDO, I. M.; SOUZA, P. D. A.; MIRANDA, C. R. L.; BORGES, E. P. Aspectos epidemiológicos das enteroparasitoses em crianças de uma unidade pública de ensino em Parnaíba, Piauí. Journal of Health Sciences, v. 16, n. 3, p. 191-196, 2014.Disponível em: $<$ https://www.researchgate.net/publication/265457455_Aspectos_Epidemiologicos_d as_Enteroparasitoses_em_Criancas_de_uma_Unidade_Publica_de_Ensino_de_Par naiba_Piaui>.

MIRANDA, P. H. S.; BEZERRA, W. F. L.; CASTRO, T. M. B. Q.; GONÇALVES, L. S. Contaminação do solo de áreas de recreação infantil de creches públicas por Ancylostoma sp. e Toxocara sp. em Teresina-PI. Revista Interdisciplinar, v. 8, n.4, p. 93-98, 2015.2 Disponível em: <https://revistainterdisciplinar.uninovafapi.edu.br/index.php/revinter/article/.../pdf_26.

OLIVEIRA, A. A. F.; FERNANDES, H. M. B.; ALCÂNTARA, N. D. F.; ASSIS, T. J. C. F.; FREITAS, F.I.S. Frequência de enteroparasitos nas areias das praias da Paraíba. Revista de Biologia e Farmácia, v. 6, n. 2, p. 108-113, 2011. Disponível em: $<$ https://docplayer.com.br/14809087-Frequencia-de-enteroparasitas-nas-areias-daspraias-da-paraiba.html>.

PRITSCH, I.C.; FRIGHETTO, M. Ocorrência de geohelmintos em areias de locais públicos municipais de videira e Itá SC, Brasil. Revista de Saúde Pública de Santa

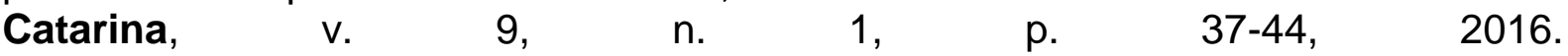
$<$ https://revista.saude.sc.gov.br/index.php/inicio/article/viewFile/375/332>.

SAITO, R. K.; RODRIGUES, A. F. S. F. A importância da análise do solo nos estudos epidemiológicos sobre parasitoses intestinais. Revista APS, v. 15, n. 2, p. 158-163, 2012. Disponível em: <https://www.escavador.com/sobre/6587901/andreflavio-soares-ferreira-rodrigues>.

SAMPAIO, J. P. S.; BARROS, V. C. Prevalência de enteroparasitoses em pacientes atendidos em uma unidade pública de saúde no município de Beneditinos - PI. Jornal Interdisciplinar de Biociências, v. 2, v. 1, p. 6-10, 2017. Disponível em: <https://doi.galoa.com.br/doi/10.17648/jibi-2448-0002-1-2-5544>. 
SILVA, A. O.; CUNHA, C. R. M.; MARTINS, W. L. L.; SILVA, L.S.; SILVA, G. R. C.; FERNANDES, C. K. C. Epidemiologia e prevenção de parasitoses intestinais em crianças das creches municipais de Itapuranga-GO. Revista faculdade Montes Belos, v. $8, \quad$ n. 1 , p. 1-17, 2015. Disponível em: <http://revista.fmb.edu.br/index.php/fmb/article/view/18>.

SOUSA, J. O.; SANTOS, E. O.; LIRA, E. M.; SÁ, I. C.; MONTEIRO, C.H. Análise parasitológica da areia das praias urbanas de João Pessoa/PB. Revista Brasileira de Ciências da Saúde, v. 18, n. 3, p. 195-202, 2014. Disponível em: <http://www.periodicos.ufpb.br/ojs/index.php/rbcs/article/view/14289>. 